\title{
A NONHOLONOMIC 3-DOF PARALLEL ROBOT
}

\author{
Patricia Ben-Horin \\ Technion - Israel Institute of Technology \\ Technion City, Haifa 32000, Israel \\ patbh@tx.technion.ac.il \\ Federico Thomas \\ Institut de Robòtica i Informàtica Industrial (CSIC-UPC) \\ 08028 Barcelona, Spain \\ fthomas@iri.upc.edu
}

\begin{abstract}
This paper addresses the problem of devising a parallel robot that can achieve a general position and orientation for its moving platform using three motors only. At first glance, this might seem impossible but, by using nonholonomic joints, three motors can manoeuver to approximate any six-degree-of-freedom motion for the platform. We analyze the case of a 3-UPU parallel robot in which each universal joint attached to the base is substituted by a spherical joint whose motion is constrained by a disk that can freely roll in contact with the sphere without slipping. The proposed robot involves fewer links and actuators than standard six-degree-of-freedom robots offering a large workspace free from leg interferences.
\end{abstract}

Keywords: Parallel robot, Nonholonomic joint, Lie bracket, underactuated robot.

\section{Introduction}

Among all possible spatial three degree-of-freedom parallel robots, those based on three UPU legs (Tsai, 1996) have drawn the attention of many researchers because of its fascinating properties. It consists of a fixed base and a moving platform connected by three serial chains, with each chain having a universal-prismatic-universal joint arranged in sequence as shown in Fig. 1(left). The universal joints are passive, only the three prismatic joints are actuated. The platform has three degrees of freedom (DOF) with respect to the base that, in general, involve coupled translations and rotations but, by properly arranging the universal joints, the moving platform can undergo pure translational [(Parenti-Castelli et al., 2000), (Di Gregorio and Parenti-Castelli, 2002)] or spherical [(Karouia and Hervé, 2000), (Di Gregorio, 2004)] motions. 

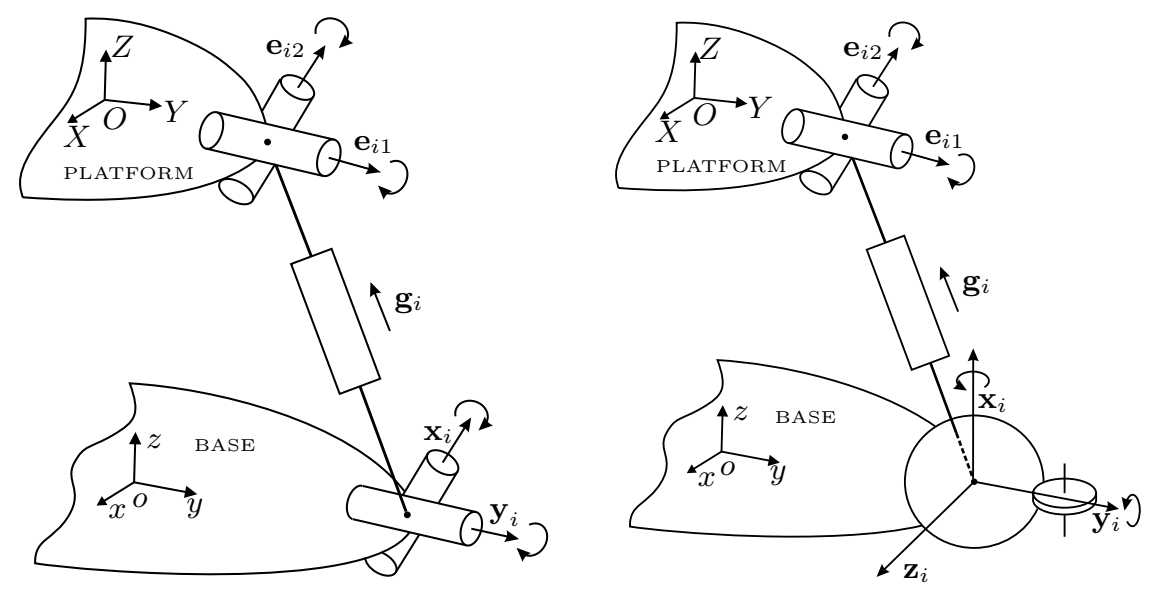

Figure 1. A UPU leg (left); and the result of substituting the universal joint attached to the base by the proposed nonholonomic joint (right).

In this paper, each universal joint attached to the base is substituted by a passive joint based on a sphere whose motion is constrained, as shown in Fig. 1(right), by a disk that can freely roll in contact with the sphere without slipping. This no-slip constraint is a nonholonomic constraint, a constraint in the velocity. The kinematics of this element is equivalent to that of a unicycle on a sphere (Hennessey, 2006). Since the disk can only roll without slipping laterally with respect to the sphere, the sphere can instantaneously only spin about $\mathbf{x}_{i}$ and $\mathbf{y}_{i}$ with respect to the base. Thus, from the instantaneous kinematics point of view, this joint is equivalent to a universal joint. The only difference being that $\mathbf{y}_{i}$ remains fixed with respect to the base, independently of the orientation of the leg. Hence, the instantaneous kinematics and statics of the resulting mechanism can be carried out using the same techniques applied to the standard 3-UPU platform (Joshi and Tsai, 2002).

There are many examples of nonholonomic systems that involve wheels rolling in contact without slipping on planes or spheres. These kind of mechanical systems have received attention because they can access a configuration space with a higher dimension than the number of its actuators. However, due to the nonintegrability of the velocity constraints, nonholonomic systems cannot follow an arbitrary path in the configuration space. Therefore, it is an important problem to find a feasible trajectory between any two configurations that satisfies the nonholonomic constraints (Li and Canny, 1993). In this paper, we concentrate ourselves on the rather simpler problem of deciding if the proposed robot 
can manoeuvre in any direction from a given configuration. This is achieved by using the concept of Lie bracket. Given two vector fields, the Lie bracket tell us if infinitesimal motions along these vector fields can be used to locally generate a motion in a direction not contained in the subspace linearly spanned by them.

To the best of our knowledge, the use of nonholonomic mechanical elements as joints in Robotics has received little attention in the past. Two related previous works are the two-motor actuated robot wrist described in (Stammers, 1993), and the continuous variable transmission described in (Moore, 1997).

This paper is structured as follows. Section 2 deals with the statics and the instantaneous kinematics of the proposed robot. Section 3 discuses the mathematical tools needed to decide if the proposed robot can manoeuvre in any direction from an arbitrary configuration. Section 4 summarizes all possible singularities for the proposed design and, finally, Section 5 offers the conclusions.

\section{Kinetostatics of the proposed robot}

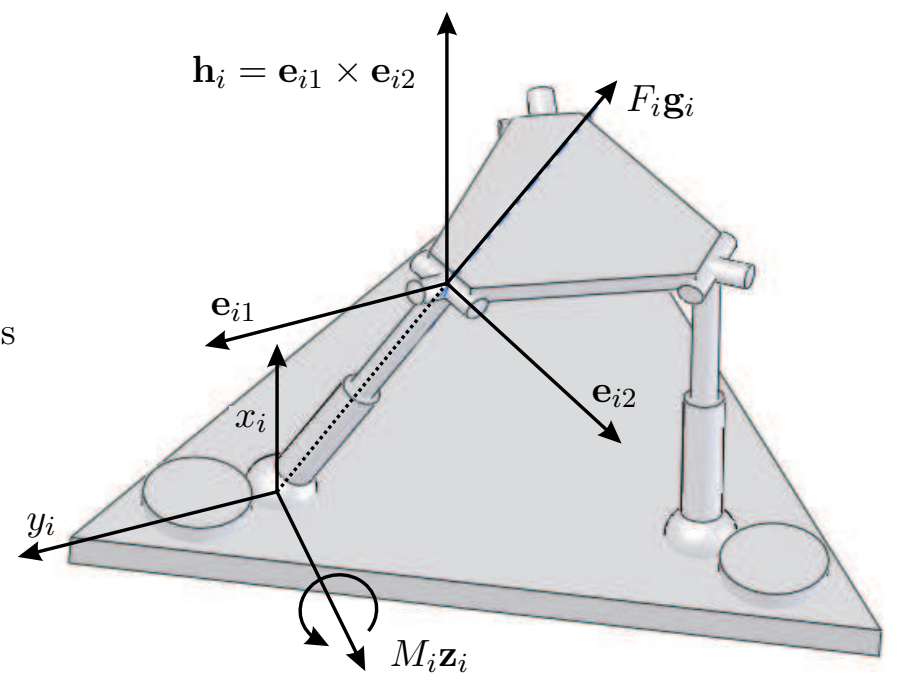

Figure 2. The proposed robot and the relevant vectors associated with its $i$ th leg, $i=1,2,3$, used in the the presented statics analysis.

In this section, we analyze the statics of a single leg, then the statics of the whole robot and, finally, its instantaneous kinematics.

Fig. 2 shows the proposed robot. In our design the universal joints attached to the platform are arranged so that the unit vectors $\mathbf{e}_{i 2}$ and 
$\mathbf{e}_{i 1}$ can be expressed as:

$$
\mathbf{e}_{i 2}=\mathbf{R}\left(\mathbf{n} \times \mathbf{r}_{i}\right), \quad \mathbf{e}_{i 1}=\mathbf{e}_{i 2} \times \mathbf{g}_{i},
$$

where $\mathbf{n}$ is the normal to the platform, $\mathbf{R}$ is the rotation matrix from the platform to the world reference frame, and $\mathbf{r}_{i}$ is the unit vector from the platform reference frame to the universal joint center. As a consequence, the vector orthogonal to both vectors can be expressed as:

$$
\mathbf{h}_{i}=\mathbf{e}_{i 1} \times \mathbf{e}_{i 2}=\left(\left(\mathbf{R}\left(\mathbf{n} \times \mathbf{r}_{i}\right)\right) \times \mathbf{g}_{i}\right) \times \mathbf{R}\left(\mathbf{n} \times \mathbf{r}_{i}\right) .
$$

As explained in the previous section, the nonholonomic joint attached to the base is constrained to only rotate instantaneously about axes $\mathbf{x}_{i}$ and $\mathbf{y}_{i}$, thus a torque between the base and the leg can only be transmitted about axis $\mathbf{z}_{i}=\mathbf{x}_{i} \times \mathbf{y}_{i}$. Likewise, a torque between the leg and the platform can only be transmitted about axis $\mathbf{h}_{i}=\mathbf{e}_{i 1} \times \mathbf{e}_{i 2}$. Then, the torque $M_{i} \mathbf{z}_{i}$ on the base is transmitted through the leg to the platform by projecting it on axis $\mathbf{h}_{i}$. In other words, the transmitted torque is $\left(M_{i} \mathbf{z}_{i} \cdot \mathbf{h}_{i}\right) \mathbf{h}_{i}$. Moreover, a force directed along the leg, say $F_{i} \mathbf{g}_{i}$, and acting at the universal joint center, generates the torque $\mathbf{R r}_{i} \times F_{i} \mathbf{g}_{i}$ on the platform.

Now, let $\mathbf{F}_{e}$ and $\mathbf{M}_{e}$ be a external force and a external torque, respectively, acting on the platform. Then, the static equilibrium equations of the platform can be written as:

$$
\begin{gathered}
\mathbf{F}_{e}=\sum_{i=1}^{3} F_{i} \mathbf{g}_{i} \\
\mathbf{M}_{e}=\sum_{i=1}^{3}\left(M_{i} \mathbf{z}_{i} \cdot \mathbf{h}_{i}\right) \mathbf{h}_{i}+\sum_{i=1}^{3} \mathbf{R r}_{i} \times F_{i} \mathbf{g}_{i}
\end{gathered}
$$

which can be rewritten in matrix form as:

$$
\begin{aligned}
& \left(\begin{array}{cccc}
\mathbf{g}_{1} & \mathbf{g}_{2} & \mathbf{g}_{3} & \ldots \\
\mathbf{R r}_{1} \times \mathbf{g}_{1} & \mathbf{R r}_{2} \times \mathbf{g}_{2} & \mathbf{R r}_{3} \times \mathbf{g}_{3}
\end{array} \cdots\right. \\
& \left.\begin{array}{lcc}
\mathbf{0}_{3 \times 1} & \mathbf{0}_{3 \times 1} & \mathbf{0}_{3 \times 1} \\
\left(\mathbf{z}_{1} \cdot \mathbf{h}_{1}\right) \mathbf{h}_{1} & \left(\mathbf{z}_{2} \cdot \mathbf{h}_{2}\right) \mathbf{h}_{2} & \left(\mathbf{z}_{3} \cdot \mathbf{h}_{3}\right) \mathbf{h}_{3}
\end{array}\right)\left(\begin{array}{c}
F_{1} \\
F_{2} \\
F_{3} \\
M_{1} \\
M_{2} \\
M_{3}
\end{array}\right)=\left(\begin{array}{c}
\mathbf{F}_{e} \\
\mathbf{M}_{e}
\end{array}\right)
\end{aligned}
$$

where the right-hand side of the above equation is known as the wrench applied on the platform. 
Finally, to obtain the linear mapping from the velocities in the prismatic actuators $\left(\dot{q}_{1}, \dot{q}_{2}, \dot{q}_{3}\right)$ to the platform linear and angular velocities $\left(\dot{x}, \dot{y}, \dot{z}, \dot{\theta_{x}}, \dot{\theta}_{y}, \dot{\theta}_{z}\right)$, that is, the platform twist, one can rely on the fact that the twist and the wrench are reciprocal to each other (Bruyninckx and Schutter, 1998). This permits to conclude that

$$
\begin{gathered}
\left(\begin{array}{c}
\dot{x} \\
\dot{y} \\
\dot{z} \\
\dot{\theta_{x}} \\
\dot{\theta_{y}} \\
\dot{\theta_{z}}
\end{array}\right)=\left(\begin{array}{cc}
\mathbf{g}_{1}^{T} & \left(\mathbf{R r}_{1} \times \mathbf{g}_{1}\right)^{T} \\
\mathbf{g}_{1}^{T} & \left(\mathbf{R r}_{1} \times \mathbf{g}_{1}\right)^{T} \\
\mathbf{g}_{1}^{T} & \left(\mathbf{R r}_{1} \times \mathbf{g}_{1}\right)^{T} \\
\mathbf{0}_{1 \times 3} & \left(\mathbf{z}_{1} \cdot \mathbf{h}_{1}\right) \mathbf{h}_{1}^{T} \\
\mathbf{0}_{1 \times 3} & \left(\mathbf{z}_{2} \cdot \mathbf{h}_{2}\right) \mathbf{h}_{2}^{T} \\
\mathbf{0}_{1 \times 3} & \left(\mathbf{z}_{3} \cdot \mathbf{h}_{3}\right) \mathbf{h}_{3}^{T}
\end{array}\right)^{-1}\left(\begin{array}{c}
\dot{q}_{1} \\
\dot{q}_{2} \\
\dot{q}_{3} \\
0 \\
0 \\
0
\end{array}\right) \\
=\left(\begin{array}{lll}
\mathbf{v}_{1} & \mathbf{v}_{2} & \mathbf{v}_{3}
\end{array}\right)\left(\begin{array}{c}
\dot{q}_{1} \\
\dot{q}_{2} \\
\dot{q}_{3}
\end{array}\right) .
\end{gathered}
$$

Note that the components of vectors $\mathbf{v}_{1}, \mathbf{v}_{2}$, and $\mathbf{v}_{3}$ are not derivatives of any generalized coordinates of the moving platform as there is no representation of the platform orientation whose derivatives correspond to angular velocities (Kim et al., 2000). Nevertheless, since $\mathbf{v}_{i}(0)=$

$\left.\frac{\partial \mathbf{x}}{\partial q_{i}}\right|_{\mathbf{x}=0}$, with $\mathbf{x}=\left(x, y, z, \theta_{x}, \theta_{y}, \theta_{z}\right)$, when analyzing the behavior of the proposed robot at a given configuration, say $\mathbf{x}_{0}$, we can always change the world reference frame so that $\mathbf{x}_{0}=\mathbf{0}$. Under this circumstance, the matrix $\left(\mathbf{v}_{1} \mathbf{v}_{2} \mathbf{v}_{3}\right)$ can be seen as an analytic jacobian.

\section{Locally reachable configurations}

The configuration of the platform can be modelled as a point in $\mathbb{R}^{3} \times$ $S O(3)$ which is locally diffeomorphic to $\mathbb{R}^{6}$, thus, equipped with a proper set of local coordinates, we are going to treat the configuration space of the platform locally as $\mathbb{R}^{6}$.

As described in the previous section, the instantaneous motion of the proposed robot can be described in terms of three vector fields $-\mathbf{v}_{1}$, $\mathbf{v}_{2}$, and $\mathbf{v}_{3}-$ associated with the three actuated prismatic joints. Linear combinations of these vector fields define a linear subspace in $\mathbb{R}^{6}$, denoted by $\operatorname{Span}\left(\mathbf{v}_{1}, \mathbf{v}_{2}, \mathbf{v}_{3}\right)$, describing all possible instantaneous motions of the platform. We would like to know the reachable set of configurations following these vector fields. While this is generally difficult globally, it is possible to learn something about the reachable set locally (Choset et al., 2005).

For two of our vector fields, say $\mathbf{v}_{1}$ and $\mathbf{v}_{2}$, consider the state reached from $\mathbf{x}_{0}$ by first following $\mathbf{v}_{1}$ for a small time $\epsilon$, then following $\mathbf{v}_{2}$ for 


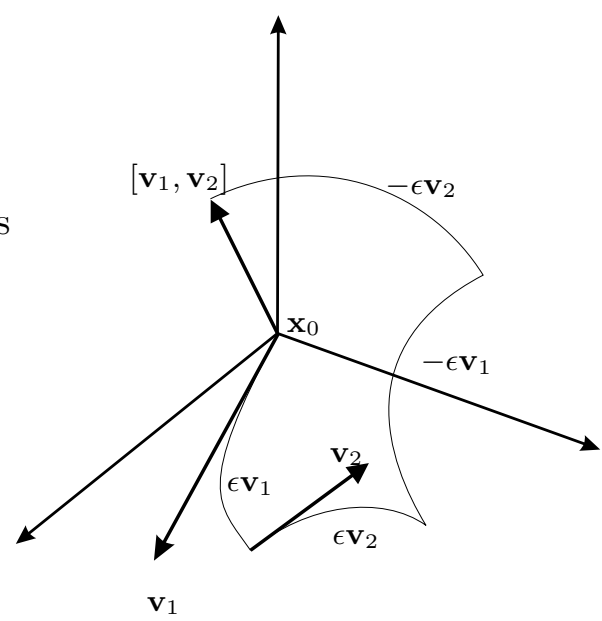

Figure 3. Geometric interpretation of the Lie bracket of the vector fields $\mathbf{v}_{1}$ and $\mathbf{v}_{2}$.

time $\epsilon$, then following $-\mathbf{v}_{1}$ for time $\epsilon$, then following $-\mathbf{v}_{2}$ for time $\epsilon$. Then, it can be shown that [see (Murray et al., 1994) p. 323]:

$$
\lim _{\epsilon \rightarrow 0} \mathbf{x}(4 \epsilon)=\mathbf{x}_{0}+\epsilon^{2}\left(\frac{\partial \mathbf{v}_{2}}{\partial \mathbf{x}} \mathbf{v}_{1}\left(\mathbf{x}_{0}\right)-\frac{\partial \mathbf{v}_{1}}{\partial \mathbf{x}} \mathbf{v}_{2}\left(\mathbf{x}_{0}\right)\right)
$$

where the partial derivatives are evaluated at $\mathbf{x}_{0}$. The term multiplying $\epsilon^{2}$ represents the net motion of the system which is technically known as the Lie bracket of $\mathbf{v}_{1}$ and $\mathbf{v}_{2}$ and denoted by $\left[\mathbf{v}_{1}, \mathbf{v}_{2}\right]$.

The Lie bracket $\left[\mathbf{v}_{1}, \mathbf{v}_{2}\right]$ defines a new vector field, and if it is not contained in $\operatorname{Span}\left(\mathbf{v}_{1}, \mathbf{v}_{2}\right)$, then it represents a new motion direction that can be followed. Locally generating motion in this direction is slower than following the vector field $\mathbf{v}_{1}$ and $\mathbf{v}_{2}$ directly, as the net motion is only $O\left(\epsilon^{3}\right)$ for time $O(\epsilon)$.

Since $\left[\mathbf{v}_{1}, \mathbf{v}_{2}\right]$ is a vector field, we can calculate its Lie bracket with another vector field, say $\mathbf{v}_{3}$. Since the resulting net motion would be $O\left(\epsilon^{9}\right)$ for time $O(\epsilon)$, these motions are not considered here for practical reasons. Hence, we limit our analysis to Lie products of degree 1, that is, $\left[\mathbf{v}_{1}, \mathbf{v}_{2}\right],\left[\mathbf{v}_{2}, \mathbf{v}_{3}\right]$, and $\left[\mathbf{v}_{3}, \mathbf{v}_{1}\right]$. Then, if the dimension of the space $\operatorname{Span}\left(\mathbf{v}_{1}, \mathbf{v}_{2}, \mathbf{v}_{3},\left[\mathbf{v}_{1}, \mathbf{v}_{2}\right],\left[\mathbf{v}_{2}, \mathbf{v}_{3}\right],\left[\mathbf{v}_{3}, \mathbf{v}_{1}\right]\right)$ is six at a given configuration $\mathbf{x}_{0}$, the robot can locally manoeuver in any direction. 


\section{Singularities}

Note that vector fields $\mathbf{v}_{1}, \mathbf{v}_{2}$, and $\mathbf{v}_{3}$ are undefined if the matrix in Eq. (2) cannot be inverted, i.e., if it is rank deficient. The configurations where this happens correspond to singularities of the robot. We can distinguish the following three cases:

(a) $\mathbf{g}_{1}, \mathbf{g}_{2}$ and $\mathbf{g}_{3}$ lie on a plane, or they are parallel to each other.

(b) $\mathbf{h}_{1}, \mathbf{h}_{2}$ and $\mathbf{h}_{3}$ lie on a plane, or any two of them are parallel.

(c) $\mathbf{h}_{i} \perp \mathbf{z}_{i}$, for $i=1,2,3$. In this case, no torque can be transmitted from the base to the platform, so that the platform gains an instantaneous degree of freedom. This corresponds to an uncertain singularity, i.e. a singularity with increased instantaneous mobility.

While the first case corresponds to a usual parallel-chain singularity, the other two correspond to singularities classified in (Zlatanov et al., 2002) as constraint singularities. These singularities are not detected when directly computing the input-output velocity equations without performing a complete statics analysis, as it has been done here.

Assuming that $\mathbf{v}_{1}, \mathbf{v}_{2}$, and $\mathbf{v}_{3}$ are well-defined, i.e., the robot is in a configuration where none of the above conditions is satisfied, nonholonomic singularities have also to be considered in our case. They are defined as those configurations in which the robot cannot manoeuvre in any direction of the configuration space. They can be characterized algebraically as those configurations satisfying

$$
\left|\begin{array}{llllll}
\mathbf{v}_{1}(\mathbf{x}) & \mathbf{v}_{2}(\mathbf{x}) & \mathbf{v}_{3}(\mathbf{x}) & {\left[\mathbf{v}_{1}(\mathbf{x}), \mathbf{v}_{2}(\mathbf{x})\right]} & {\left[\mathbf{v}_{2}(\mathbf{x}), \mathbf{v}_{3}(\mathbf{x})\right]} & {\left[\mathbf{v}_{3}(\mathbf{x}), \mathbf{v}_{1}(\mathbf{x})\right]}
\end{array}\right|=0 .
$$

The explicit computation of the above expression requires a computer algebra system. It remains to be seen how these singularities are distributed in the working space of the robot.

\section{Conclusions}

A car-like robot cannot move sideways but, by manoeuvring, it can reach any place and orientation on a plane. In this paper, by introducing nonholonomic joints, we have extended this behavior to parallel robots. This permits reducing the number of actuators, and hence the complexity of the robot, at the cost of motion speed.

We have analyzed the case of a 3-UPU parallel robot in which each universal joint attached to the base has been substituted by a nonholonomic joint whose instantaneous kinematics is equivalent to that of a universal joint. Many other architectures can be devised based on this substitution. 


\section{Acknowledgements}

This work has been partially supported by the Spanish Ministry of Education and Science through contract DPI2007-60858, and by the Research Network on Advanced Production Techniques of the Autonomous Government of Catalonia (Xartap).

\section{References}

Bruyninckx, H., and De Schutter, J. (1998), Unified Kinetostatics for serial, parallel and mobile robots, Proceedings of ARK'98, Strobl, Austria.

Choset, H., Lynch, K.M., Hutchinson, S., Kantor, G., Burgard, W., Kavraki, L.E., and Thrun, S. (2005), Principles of Robot Motion: Theory, Algorithms, and Implementations, MIT Press, Boston.

Di Gregorio, R., and Parenti-Castelli, V. (2002), Mobility analysis of the 3-UPU parallel mechanism assembled for pure translational motion, ASME Journal of Mechanical Design, vol. 124, pp. 259-264, 2002.

Di Gregorio, R. (2004), Statics and singularity loci of the 3-UPU wrist, IEEE Trans. on Robotics, vol. 30, no. 4, pp. 630-635.

Hennessey, M.P. (2006), Visualizing the motion of a unicycle on a sphere, Int. J. of Modelling and Simulation, vol. 26, no. 1, pp. 69-79.

Huang, Z., Li, S.H., and Zuo, R.G. (2004), Feasible instantaneous motions and kinematic characteristics of a special 3-DOF 3-UPU parallel manipulator, Mechanism and Machine Theory, vol. 39, pp. 957-970.

Joshi, S.A., and Tsai, L-W. (2002), Jacobian Analysis of Limited-DOF Parallel Manipulators, Trans. of the ASME, vol. 124, pp. 254-258.

Karouia, M., and Hervé, J.M. (2000), A three-dof tripod for generating spherical rotation, Proceedings of ARK'00, Portoroz, Slovenia.

Kim, D., Chung, W., and Youm, Y. (2000), Analytic jacobian of in-parallel manipulators, Proceedings of IEEE ICRA'00, San Fancisco, USA.

Li, Z., and Canny, J.F. (Eds.) (1993), Nonholonomic motion planning, Kluwer Academic Publishers, Boston.

Moore, C.A. (1997), Continuously variable transmission for serial link cobot architectures, Master Thesis, Nothwestern University.

Murray, R.M., Li, Z., and Sastry, S.S. (1994), A Mathematical introduction to robotic manipulation, CRC Press, Boca Raton.

Parenti-Castelli, V., Di Gregorio, R., and Rubani, F. (2000), Workspace and optimal design of a pure translation parallel manipulator, Meccanica, vol. 35, pp. 203-214.

Stammers, C.W. (1993), Operation of a two-motor robot wrist to achive three-dimensional manoeuvres with minimum total rotation, Proc. Inst. Mech. Eng., C, vol. 207, no. 1, pp. 33-39.

Tsai, L-W. (1996), Kinematics of a three-DOF platform with three extensible limbs, Proceedings of ARK'96, Portoroz-Bernandin, Slovenia.

Tsai, L-W., and Joshi, S.A. (2000), Kinematics and optimization of a spatial 3-UPU parallel manipulator, ASME Journal of Mechanical Design, vol. 122, pp. 439-446.

Zlatanov, D., Bonev, I.A., Gosselin, C.M. (2002), Constraint Singularities of parallel Mechanisms, Proceedings of the IEEE ICRA, Washington, DC, USA. 